\title{
Agro-morphological Characteristics and Sensory Evaluation of Native Peruvian Chili Peppers
}

\author{
Kirti Patel ${ }^{1}$, Rosa Calderon ${ }^{1}$, Edgard Asencios ${ }^{1}$, Dioliza Vilchez ${ }^{2}$, Mavel Marcelo ${ }^{2}$ and Rosario Rojas ${ }^{1}$ \\ 1. Unidad de Investigación en Productos Naturales, Laboratorios de Investigación y Desarrollo, Facultad de Ciencias y Filosofía, \\ Universidad Peruana Cayetano Heredia, Lima 31, Perú \\ 2. Sub Dirección de Recursos Genéticos, Dirección de Recursos Genéticos y Biotecnología, Instituto Nacional de Innovación \\ Agraria, Lima 12, Perú
}

\begin{abstract}
In this work, 31 native Peruvian chili peppers were evaluated for their agro-morphological characteristics and sensory attributes. The descriptive sensory analysis (DSA) was used to evaluate the 31 chili pepper samples based on their acid, bell pepper, sweet, tomato, apple, citrus, fruity, herbal, oregano and passion fruit attributes. The data obtained from the DSA enabled the grouping of these 31 chili peppers into six groups based on their different sensory attributes, such as fruity, bell pepper and herbal. The most abundant group (12 of the 31) had the bell pepper, apple, herbal, fruity and sweet attributes. Each group contained different species and different physical appearances, indicating that species or different forms of chili pepper do not define that sensory attribute. Considering the fact that Peruvian peppers are in high demand, the results achieved would be useful for growers, producers and chefs, as well as for further breeding activities.
\end{abstract}

Key words: Chili peppers, Peru, sensory analysis, agro-morphological characteristics.

\section{Introduction}

Peru possesses the highest diversity of cultivated chili peppers in the world and is one of the few countries where all five domesticated species are cultivated and used in local diet [1]. Peruvian chili peppers with their great diversity have an increasing popularity internationally; especially, a rise in Andean style restaurants has led to the need of research in understanding their flavour and aroma.

There are limited studies on Peruvian chili peppers. One paper reports the effect of different environments on the chemical attributes, indicating that due to the change in meteorological conditions and agricultural practices, there was a difference in the phytochemical profiles [2]. A study on 147 dry pepper samples from Peru quantified their content of flavonoids, fat vitamin $\mathrm{C}$ and extractable color [1]. Another study on 32 different accessions of native Peruvian Capsicum

Corresponding author: Rosario Rojas, Ph.D., research field: natural products. pubescens (Rocoto) concluded that their phytochemical composition was less diverse, as compared to other four Peruvian domesticated Capsicum species [3]. A most recent study on Peruvian chili peppers showed the volatile composition of 50 fresh samples, which were clustered into groups based on hydrocarbons, esters, terpenes, aldehyde and ketones composition [4].

Very few studies have reported the sensory attributes that contribute to the flavor of Capsicum species. For green bell peppers (C. апnиum), grassy, cucumber and green bell pepper attributes were reported; whereas red bell peppers were reported to have sweet, sour and red bell pepper aroma attributes [5]. Analysis of C. chinense from Brazil showed high pepper-like and green-note compounds [6]. Two Andean species of chili peppers, C. baccatum (Ají) and C. pubescens (Rocoto), had an earthy/vegetable/bell pepper like aroma with a distinct cucumber odor [7]. Sensory analysis showed that elite sweet pepper (C. annuum) lines and hybrids from a 
commercial breeding program in Netherlands had sensory attributes such as sweet and fruity apple [8].

To our knowledge, there is no information on fresh Peruvian chili peppers regarding their flavor. Considering the increase in their popularity, especially to gastronomy, it is important to understand their unique flavor and sensory attributes. Therefore, the main purpose of this study was to evaluate the agro-morphological characteristics and sensory properties of 31 Peruvian chili peppers, as well as to group the chili peppers based on their sensory evaluation.

The overall outcome of this research could be a starting point towards the directed flavor breeding in chili peppers. The results highlight the individual flavor and sensory attributes of Peruvian chili peppers and this may lead to a further increase in their demand in the international markets.

\section{Materials and Methods}

\subsection{Chili Pepper Samples}

Chili peppers samples were collected in January and February 2015 from four locations representing the different environments where chili peppers are grown, namely, (1) a farm located in a gorge of Grocio Prado district in Chincha province (dry flat area), (2) a farm in the city of Pucallpa in Coronel Portillo province (mountain wet area), (3) the agricultural station of Instituto Nacional de Innovacion Agraria (INIA) located in Huaral, North Lima (dry flat area) and (4) C. pubescens "Rocoto" samples were collected from the department of Pasco in the district of Villa Rica (forest region).

Agro-morphological characterization was performed using descriptors for the plant and fruit, as established by the International Plant Genetic Resources Institute (IPGRI) [9]. Habit of growth was observed for each variety at the time of start of first fruit ripening in $50 \%$ of growing plants. Plant height and the number and total weight of fruits per plant were calculated as the average of data obtained from five samples. Each fruit is described based on their color, shape, length, diameter and weight observed, when each of them reached full physiological ripeness. Performance was expressed as the weight of fruit per hectare.

\subsection{Descriptive Sensory Analysis (DSA)}

The 31 fresh chili pepper samples were evaluated by five trained panelists. Training of the candidates for a 10-month duration resulted in a selection of five final panelists from 75 candidates, according to the procedures given by the NTP-ISO 6564 [10] and NTP-ISO 6658 [11]. Panelists' selection criteria were based on pepper eating habits, sensibility to basic tastes and smells, as well as individual reproducibility and degree of agreement with the rest of the members. Four flavor (acid, bell pepper, sweet and tomato) and six aroma (apple, citrus, fruity, herbal, oregano and passion fruit) descriptors, as well as references for lower and upper limits, were defined by consensus. All tests were carried out in individual booths under controlled lighting (white, green, red and purple) and temperature $\left(22{ }^{\circ} \mathrm{C}\right)$. Whole peppers were given in plastic cups coded with three-digit random numbers, and each sample was analyzed three times in different sessions. Cold water and cream crackers were served to eliminate aftertaste and reduce pungency. First, the panelists analyzed aroma by making a longitudinal cut of the fruit, then flavor was assessed by cutting a piece of pericarp. All impressions were registered on a horizontal $10 \mathrm{~cm}$ line scale marking near the lower or upper limit depending on whether the sample was more similar to each respective reference.

\subsection{Statistical Analysis}

Data from the sensory analysis of chili pepper samples are reported as mean $(n=3)$. Differences were considered to be significant at $P \leq 0.05$. Data were analyzed by one way ANOVA (sample) using XLSTAT (version 2015.3.01, Addinsoft SARI, Paris, France). 


\section{Results and Discussion}

The agronomical information of the 31 chili peppers studied is shown in Table 1 and Fig. 1. The fruit morphological features, such as color, fruit form and size, were determined on five ripe fruits. In all accessions, the fruit color at the ripe stage was red, but the level of red varied from dark red, e.g., in "Ají Montaña" (1: C. chinense) to clear red, such as in "Ají Dulce Rojo" (72: C. chinense). Several accessions had fruit with special colors (Fig. 1), such as orange (193), brown (354), lemon yellow (10ica) and yellow orange (13p). The fruit shapes and sizes varied in the samples studied. Based on the criteria, they can be grouped in different types, such as bell shaped, triangular, almost round and elongated. The bell shaped fruit type generally had similar fruit length and diameter. Several samples had an almost round shape (12p, 13p and 1 ica) and all belonged to $C$. frutescens. There was a great diversity in fruit length $(6-122 \mathrm{~mm})$, fruit diameter (6-64 mm), fruit weight (0.3-90.2 g), number of fruits per plant (16-905) and plant height (27-183 $\mathrm{cm})$.

Table 1 Agro-morphological characteristics of 31 Peruvian chili peppers.

\begin{tabular}{|c|c|c|c|c|c|c|c|c|c|}
\hline Code & Species & Accession name & Color & Fruit form & $\begin{array}{l}\text { Length } \\
(\mathrm{mm})\end{array}$ & $\begin{array}{l}\text { Diameter } \\
(\mathrm{mm})\end{array}$ & $\begin{array}{l}\text { Fruit } \\
\text { weight (g) }\end{array}$ & $\begin{array}{l}\text { No. of } \\
\text { fruits } \\
\text { per plant }\end{array}$ & $\begin{array}{l}\text { Plant } \\
\text { height } \\
(\mathrm{cm})\end{array}$ \\
\hline 1 & C. chinense & Aji Montaña & Dark red & Bell shaped & 33 & 23 & 5.8 & 157 & 126 \\
\hline 7 & C. baccatum & Tomatito Rojo & Red & Triangular & 29 & 22 & 4.5 & 52 & 27 \\
\hline 10 & C. baccatum & Aji Limo Rojo & Dark red & Triangular & 56 & 28 & 14.1 & 107 & 28 \\
\hline 18 & C. baccatum & Puca Limo & Dark red & Triangular & 56 & 28 & 14.1 & 117 & 28 \\
\hline 29 & C. frutescens & Aji Pipi Rojo & Red & Elongated & 24 & 6 & 0.3 & 615 & 144 \\
\hline 38 & C. chinense & Picante Rojo & Red & Bell shaped & 28 & 20 & 3.8 & 212 & 142 \\
\hline 40 & C. chinense & Aji Dulce Rojo & Red & Bell shaped & 38 & 18 & 4.7 & 227 & 134 \\
\hline 72 & C. chinense & Aji Dulce Rojo & Clear red & Elongated & 39 & 11 & 2.2 & 544 & 119 \\
\hline 175 & C. baccatum & Aji Uña de Gavilan & Red & Elongated & 64 & 20 & 7.5 & 80 & 77 \\
\hline 184 & C. annuum & Aji Cerezo & Dark red & Triangular & 26 & 26 & 8.7 & 16 & 32 \\
\hline 191 & C. frutescens & Aji Pinchito Rojo & Clear red & Elongated & 21 & 7 & 0.5 & 905 & 138 \\
\hline 193 & C. baccatum & Escabeche & Orange & Elongated & 104 & 27 & 21.4 & 28 & 125 \\
\hline 203 & C. chinense & Aji Rojo & Dark red & Triangular & 23 & 12 & 3.3 & 185 & 134 \\
\hline 339 & С. annuum & Aji Macruzori Picante & Red & Elongated & 98 & 10 & 1.6 & 163 & 119 \\
\hline 354 & C. chinense & Aji Panca & Brown & Elongated & 80 & 25 & 14.0 & 59 & 100 \\
\hline 374 & C. frutescens & Asna Uchu & Clear red & Elongated & 26 & 9 & 0.8 & 440 & 183 \\
\hline 377 & C. chinense & Asna Uchu Grande & Dark red & Elongated & 95 & 25 & 19.9 & 72 & 123 \\
\hline 401 & C. frutescens & Aji Mono & Red & Triangular & 24 & 11 & 1.3 & 92 & 168 \\
\hline 10ica & C. chinense & Miscucho & Lemon yellow & Triangular & 51 & 29 & 10.3 & 56 & 106 \\
\hline 11 ica & C. chinense & $\begin{array}{l}\text { Corazon de Paloma } \\
\text { Rojo }\end{array}$ & Red & Triangular & 21 & 13 & 1.6 & 89 & 43 \\
\hline $12 p$ & C. frutescens & Charapita Rojo & Red & Almost round & 8 & 10 & 0.4 & 111 & 117 \\
\hline $13 p$ & C. frutescens & Charapita Amarillo & Yellow orange & Almost round & 6 & 9 & 0.3 & 127 & 106 \\
\hline $16 \mathrm{p}$ & C. chinense & Pucunucho & Yellow orange & Triangular & 50 & 17 & 3.2 & 70 & 75 \\
\hline 1ica & C. frutescens & Charapon & Red & Almost round & 14 & 16 & 1.2 & 39 & 131 \\
\hline $1 \mathrm{vr}$ & C. pubescens & Rocoto Rojo & Red & Bell shaped & 55 & 50 & 53.6 & 34 & 169 \\
\hline $2 \mathrm{vr}$ & C. pubescens & Rocoto Amarillo & Pale yellow orange & Bell shaped & 75 & 64 & 90.2 & 26 & 156 \\
\hline 4 ica & C. baccatum & Aji Amarillo & Yellow orange & Elongated & 122 & 32 & 38.8 & 27 & 122 \\
\hline 5 ica & C. baccatum & Ayucllo & Red & Bell shaped & 33 & 17 & 3.7 & 45 & 86 \\
\hline 6ica & C. baccatum & Challuaruro & Clear red & Elongated & 58 & 13 & 3.4 & 162 & 65 \\
\hline 7ica & C. chinense & Limo & Red & Triangular & 58 & 33 & 9.5 & 81 & 86 \\
\hline 9ica & C. chinense & $\begin{array}{l}\text { Corazon de Paloma } \\
\text { Amarillo }\end{array}$ & Yellow orange & Triangular & 21 & 14 & 1.6 & 78 & 36 \\
\hline
\end{tabular}




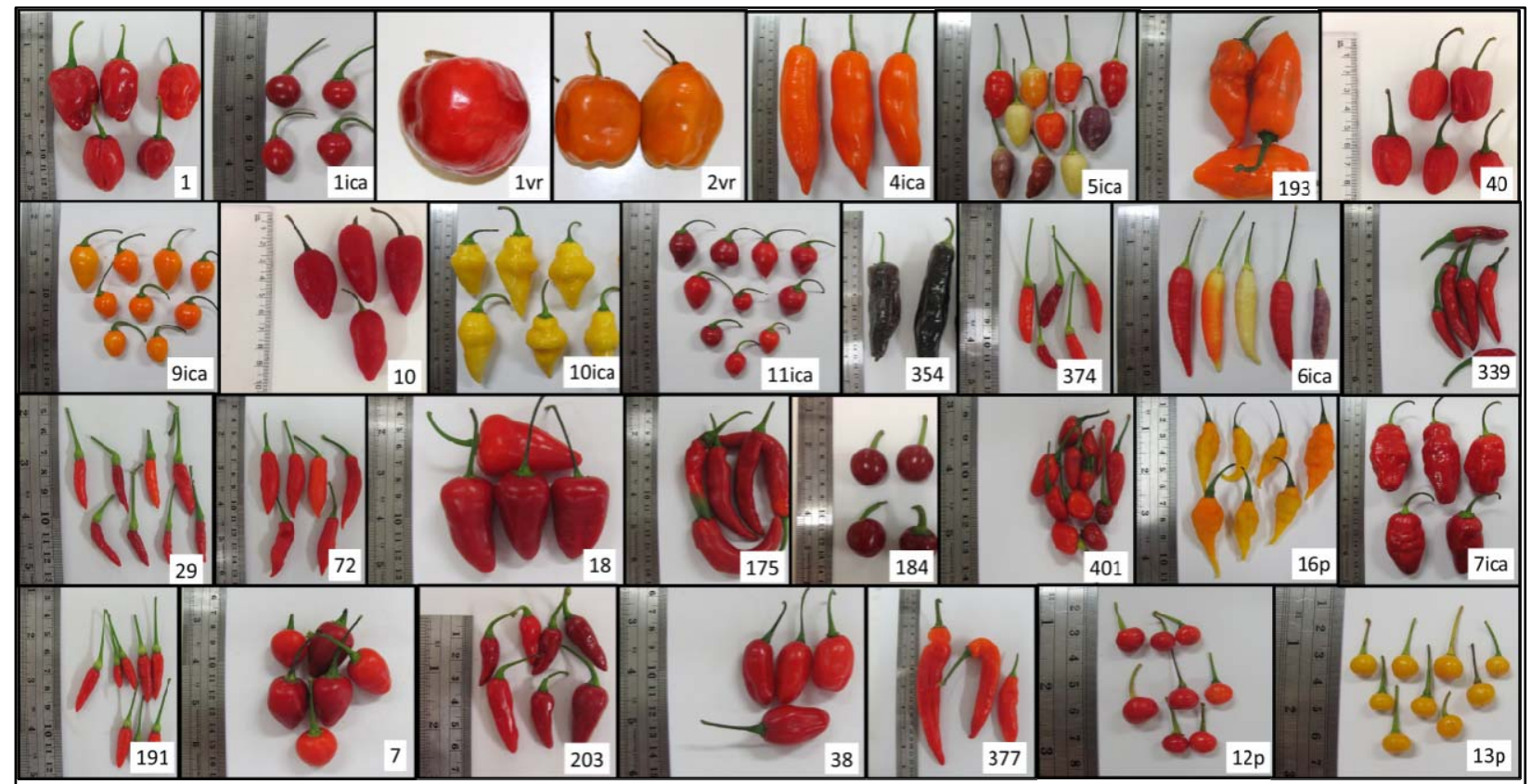

Fig. 1 Representative variation in fruit color and shape among 31 chili peppers.

The numbers in this figure corresponds with code of different chili peppers.

The sensory quality of different cultivars of chili peppers was evaluated through DSA. The panelists identified a total of four flavor (acid, bell pepper, sweet and tomato) and six aroma (apple, citrus, fruity, herbal, oregano and passion fruit) attributes. Citrus attribute was described by tangerine, orange and lime references, while fruity attribute was described by pear, banana, papaya, apricot, pineapple and mango references. Oregano attribute was different from the herb attribute; the reference for the latter was grass, chamomile, lemon verbena, mint and parsley. The attribute acidity was referenced by green pepper, watermelon and green and red apple. Aji Amarillo ( $C$. baccatum) and bell pepper were the reference for the sweet attribute. The average mean results for the sensory analysis of 31 chili peppers are shown in Table 2. Significant differences among the 31 chili peppers were established for all the 10 attributes (Table 3). All sensory attributes were highly significantly different $(P<0.001)$ among the chili peppers samples. The 31 chili pepper samples were relatively dispersed, indicating variation in terms of sensory profiles, as shown in Fig. 2 and Table 4. Group A (9ica, 13p and 401) was perceived to be higher in fruity, passion fruit and citrus notes. Group C (16p, 374 and 191) also perceived a fruit note, with a high score on the apple descriptor. Group B (354 and 29) was differentiated by herbal and oregano notes. Group D (4ica, 38, 175, 1 and 7), group E (2vr, 1vr, 184, 339, 203 and 377) and group F (7ica, 18, 40, 1ica, 5ica, 11ica, 6ica, 12p, 72, 10ica, 193 and 10) all had a bell pepper note presence; but group D has a sweet note, group $\mathrm{E}$ had a herbal note and group $\mathrm{F}$ had an apple, herbal, sweet and fruity note.

On the basis of the sensory results (Tables 2-4 and Fig. 2), the sensory profiles of these 31 chili peppers can be grouped into three large categories broadly related to fruity (group A and C), herbal (group B) and bell pepper (group D, E and F). The 31 chili pepper samples (Groups A-F) are comprised of several different chili peppers species and physical appearances (Table 4 and Fig. 2), suggesting that species or physical forms of chili peppers do not govern sensory attributes. 
Table 2 Mean scores of sensory descriptors of 31 chili pepper samples.

\begin{tabular}{|c|c|c|c|c|c|c|c|c|c|c|c|c|}
\hline \multirow[b]{2}{*}{ Code } & \multirow[b]{2}{*}{ Species } & \multirow[b]{2}{*}{ Accession name } & \multicolumn{10}{|c|}{ Sensory descriptors ${ }^{*}$} \\
\hline & & & Acid & Citrus & Sweet & Fruity & Herbal & Apple & $\begin{array}{l}\text { Passion } \\
\text { fruit }\end{array}$ & Oregano & $\begin{array}{l}\text { Bell } \\
\text { pepper }\end{array}$ & Tomato \\
\hline 1 & C. chinense & Aji Montaña & 2.51 & 3.47 & 6.61 & 5.91 & 5.71 & 6.09 & 2.67 & 3.84 & 5.59 & 3.43 \\
\hline 7 & C. baccatum & Tomatito Rojo & 2.79 & 3.97 & 8.99 & 5.67 & 5.14 & 4.22 & 2.97 & 3.64 & 6.32 & 3.63 \\
\hline 10 & C. baccatum & Aji Limo Rojo & 3.65 & 4.25 & 5.80 & 4.77 & 5.91 & 3.84 & 3.18 & 4.48 & 5.81 & 4.07 \\
\hline 18 & C. baccatum & Puca Limo & 3.17 & 4.47 & 6.24 & 6.27 & 5.52 & 4.79 & 3.48 & 3.79 & 7.33 & 4.34 \\
\hline 29 & C. frutescens & Aji Pipi Rojo & 3.57 & 2.08 & 1.59 & 4.03 & 4.13 & 3.11 & 1.09 & 3.99 & 2.97 & 1.65 \\
\hline 38 & C. chinense & Picante Rojo & 3.46 & 7.63 & 7.16 & 5.61 & 4.56 & 4.17 & 2.22 & 3.15 & 6.33 & 4.90 \\
\hline 40 & C. chinense & Aji Dulce Rojo & 3.00 & 4.86 & 6.11 & 6.16 & 4.72 & 4.76 & 4.17 & 3.69 & 6.36 & 4.81 \\
\hline 72 & C. chinense & Aji Dulce Rojo & 3.46 & 3.37 & 3.96 & 6.14 & 5.47 & 4.97 & 2.79 & 3.68 & 6.56 & 4.45 \\
\hline 175 & C. baccatum & Aji Uña de Gavilan & 2.34 & 3.53 & 6.09 & 5.43 & 4.92 & 4.31 & 2.00 & 2.66 & 6.20 & 3.72 \\
\hline 184 & C. annuum & Aji Cerezo & 3.59 & 2.33 & 4.44 & 4.17 & 3.67 & 2.48 & 0.75 & 2.19 & 6.09 & 3.27 \\
\hline 191 & C. frutescens & Aji Pinchito Rojo & 4.56 & 3.42 & 1.61 & 5.99 & 5.34 & 4.87 & 2.09 & 2.92 & 1.89 & 1.21 \\
\hline 193 & C. baccatum & Escabeche & 2.87 & 4.24 & 5.83 & 5.42 & 5.70 & 5.00 & 3.51 & 3.84 & 4.27 & 3.74 \\
\hline 203 & C. chinense & Aji Rojo & 2.27 & 3.37 & 5.19 & 4.97 & 4.85 & 3.81 & 1.83 & 4.20 & 4.60 & 1.72 \\
\hline 339 & C. апnиит & Aji Macruzori Picante & 3.70 & 3.36 & 3.23 & 3.85 & 5.26 & 2.37 & 2.09 & 3.96 & 4.55 & 2.35 \\
\hline 354 & C. chinense & Aji Panca & 2.51 & 1.75 & 2.40 & 4.29 & 5.91 & 3.43 & 0.45 & 3.95 & 3.91 & 4.09 \\
\hline 374 & C. frutescens & Asna Uchu & 3.24 & 4.80 & 2.43 & 7.48 & 4.89 & 6.43 & 2.07 & 4.89 & 2.21 & 2.03 \\
\hline 377 & C. chinense & Asna Uchu Grande & 1.37 & 1.25 & 5.95 & 4.25 & 5.95 & 3.45 & 1.00 & 4.12 & 6.25 & 3.12 \\
\hline 401 & C. frutescens & Aji Mono & 3.37 & 4.33 & 2.77 & 7.97 & 5.23 & 5.70 & 5.40 & 3.70 & 3.17 & 2.73 \\
\hline 10ica & C. chinense & Miscucho & 3.12 & 4.54 & 4.80 & 5.39 & 4.70 & 4.16 & 4.11 & 3.17 & 4.14 & 3.72 \\
\hline 11 ica & C. chinense & Corazon de Paloma Rojo & 4.51 & 5.51 & 5.35 & 6.81 & 4.43 & 4.81 & 3.33 & 3.81 & 5.81 & 3.91 \\
\hline $12 \mathrm{p}$ & C. frutescens & Charapita Rojo & 3.38 & 4.21 & 3.80 & 6.32 & 4.36 & 4.31 & 3.36 & 2.61 & 5.01 & 3.38 \\
\hline $13 p$ & C. frutescens & Charapita Amarillo & 3.66 & 4.95 & 2.57 & 6.24 & 4.98 & 4.54 & 4.54 & 3.35 & 2.40 & 2.54 \\
\hline $16 p$ & C. chinense & Pucunucho & 2.25 & 4.32 & 2.96 & 5.61 & 3.82 & 4.20 & 3.00 & 1.96 & 2.84 & 2.55 \\
\hline 1ica & C. frutescens & Charapon & 3.38 & 4.98 & 5.04 & 6.76 & 5.39 & 4.28 & 4.11 & 3.77 & 6.52 & 3.37 \\
\hline $1 \mathrm{vr}$ & C. pubescens & Rocoto Rojo & 2.12 & 2.45 & 3.79 & 3.63 & 3.98 & 3.51 & 0.27 & 1.99 & 3.51 & 3.15 \\
\hline $2 \mathrm{vr}$ & C. pubescens & Rocoto Amarillo & 2.75 & 2.91 & 5.10 & 4.63 & 4.24 & 3.79 & 0.91 & 1.86 & 4.45 & 3.55 \\
\hline 4ica & C. baccatum & Aji Amarillo & 3.15 & 5.72 & 7.92 & 7.76 & 4.25 & 4.90 & 4.92 & 2.60 & 5.35 & 4.49 \\
\hline 5 ica & C. baccatum & Ayucllo & 3.63 & 4.86 & 5.19 & 6.62 & 4.06 & 5.50 & 2.58 & 2.81 & 5.42 & 3.90 \\
\hline 6ica & C. baccatum & Challuaruro & 4.11 & 4.26 & 3.99 & 6.49 & 4.37 & 4.67 & 3.13 & 2.59 & 5.41 & 4.07 \\
\hline 7ica & C. chinense & Limo & 3.24 & 4.52 & 5.58 & 6.10 & 4.95 & 5.28 & 2.68 & 3.08 & 7.18 & 4.62 \\
\hline 9ica & C. chinense & $\begin{array}{l}\text { Corazon de Paloma } \\
\text { Amarillo }\end{array}$ & 3.25 & 5.40 & 4.89 & 6.85 & 4.89 & 4.42 & 6.21 & 2.92 & 4.31 & 3.49 \\
\hline
\end{tabular}

${ }^{*}$ Scores of sensory descriptors were evaluated on a 0-10 scale.

Table 3 Results of sensory descriptive analysis for 31 chili peppers.

\begin{tabular}{lllll}
\hline Attribute & Minimum & Maximum & Mean & SD \\
\hline Acid & 1.37 & 4.56 & 3.16 & 0.69 \\
Citrus & 1.25 & 7.63 & 4.04 & 1.29 \\
Sweet & 1.59 & 8.99 & 4.75 & 1.79 \\
Fruity & 3.63 & 7.97 & 5.73 & 1.14 \\
Herbal & 3.67 & 5.95 & 4.88 & 0.65 \\
Apple & 2.37 & 6.43 & 4.39 & 0.93 \\
Passion fruit & 0.27 & 6.21 & 2.80 & 1.44 \\
Oregano & 1.86 & 4.89 & 3.33 & 0.77 \\
Bell pepper & 1.89 & 7.33 & 4.93 & 1.51 \\
Tomato & 1.21 & 4.90 & 3.42 & 0.95 \\
\hline
\end{tabular}

SD: standard deviation. Scores of sensory descriptors were evaluated on a 0-10 scale. Differences among sensory attributes were statistically significant $(P<0.001)$. 

Native Peruvian Chili Peppers

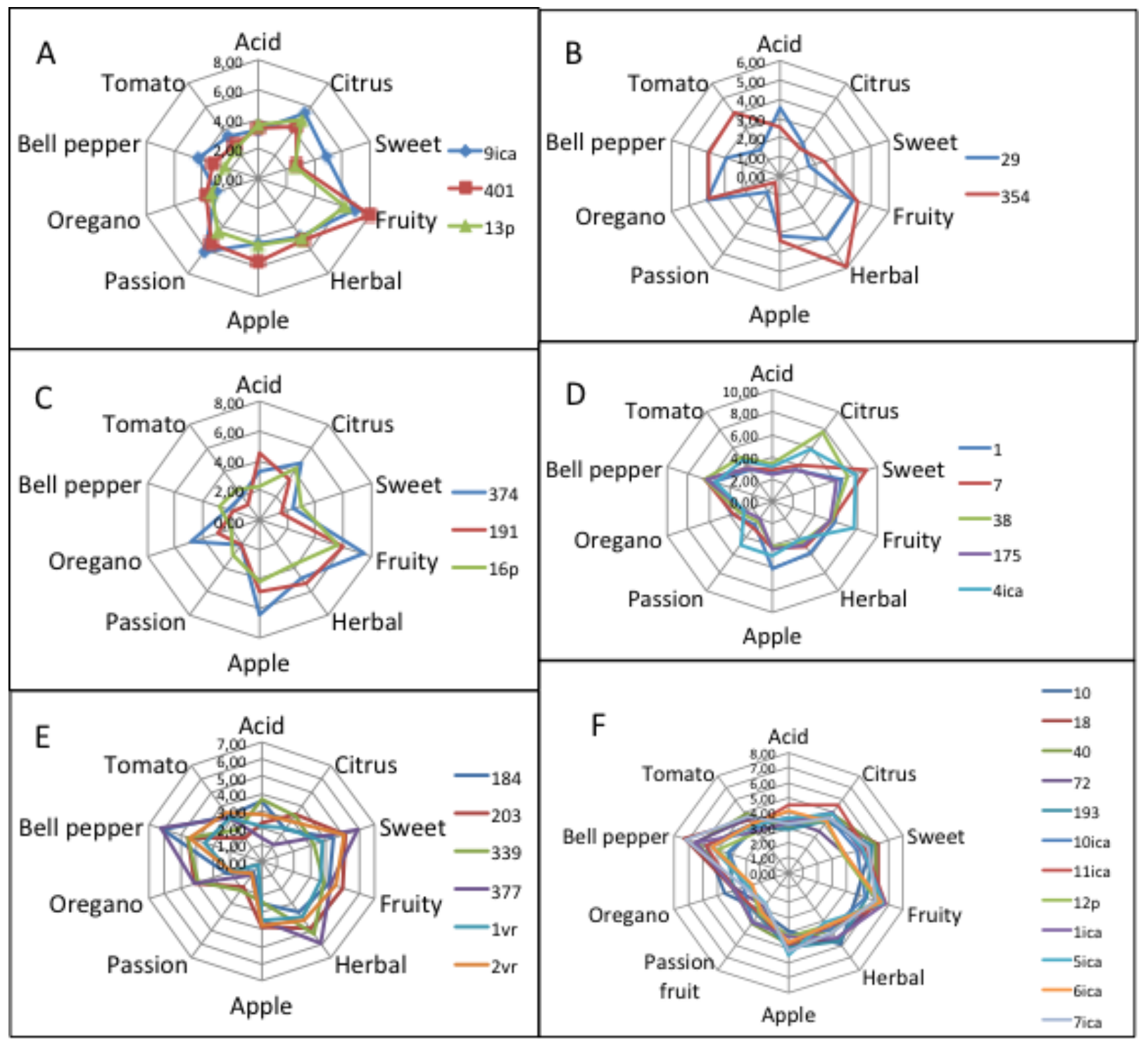

Fig. 2 Descriptive sensory analysis of 31 chili peppers and grouping according to their sensory profiles.

Table 4 Sensory analysis and morphological characteristics of different chili pepper groups.

\begin{tabular}{|c|c|c|c|c|c|c|}
\hline Group & Sensory attribute & Code & Species & Accession name & Color & Fruit form \\
\hline \multirow{3}{*}{ A } & \multirow{3}{*}{$\begin{array}{l}\text { Fruity, passion fruit, } \\
\text { citrus }\end{array}$} & 9ica & C. chinense & $\begin{array}{l}\text { Corazon de Paloma } \\
\text { Amarillo }\end{array}$ & Yellow orange & Triangular \\
\hline & & 401 & C. frutescens & Aji Mono & Red & Triangular \\
\hline & & $13 p$ & C. frutescens & Charapita Amarillo & Yellow orange & Almost round \\
\hline \multirow{2}{*}{ B } & \multirow{2}{*}{ Herbal, oregano } & 29 & C. frutescens & Aji Pipi Rojo & Red & Elongated \\
\hline & & 354 & C. chinense & Aji Panca & Brown & Elongated \\
\hline \multirow{3}{*}{$\mathrm{C}$} & \multirow{3}{*}{ Fruit, apple } & 374 & C. frutescens & Asna Uchu & Clear red & Elongated \\
\hline & & 191 & C. frutescens & Aji Pinchito Rojo & Clear red & Elongated \\
\hline & & $16 \mathrm{p}$ & C. chinense & Pucunucho & Yellow orange & Triangular \\
\hline \multirow{5}{*}{$\mathrm{D}$} & \multirow{5}{*}{ Bell pepper, sweet } & 1 & C. chinense & Aji Montaña & Dark red & Bell shaped \\
\hline & & 7 & C. baccatum & Tomatito Rojo & Red & Triangular \\
\hline & & 38 & C. chinense & Picante Rojo & Red & Bell shaped \\
\hline & & 175 & C. baccatum & Aji Uña de Gavilan & Red & Elongated \\
\hline & & 4 ica & C. baccatum & Aji Amarillo & Yellow orange & Elongated \\
\hline
\end{tabular}




\begin{tabular}{|c|c|c|c|c|c|c|}
\hline Group & Sensory attribute & Code & Species & Accession name & Color & Fruit form \\
\hline \multirow{6}{*}{$\mathrm{E}$} & \multirow{6}{*}{ Bell pepper, herbal } & 184 & C. annuum & Aji Cerezo & Dark red & Triangular \\
\hline & & 203 & C. chinense & Aji Rojo & Dark red & Triangular \\
\hline & & 339 & C. апnиит & Aji Macruzori Picante & Red & Elongated \\
\hline & & 377 & C. chinense & Asna Uchu Grande & Dark red & Elongated \\
\hline & & $1 \mathrm{vr}$ & C. pubescens & Rocoto Rojo & Red & Bell shaped \\
\hline & & $2 \mathrm{vr}$ & C. pubescens & Rocoto Amarillo & Pale yellow orange & Bell shaped \\
\hline \multirow{12}{*}{$\mathrm{F}$} & \multirow{12}{*}{$\begin{array}{l}\text { Bell pepper, apple, } \\
\text { herbal, fruity, sweet }\end{array}$} & 10 & C. baccatum & Aji Limo Rojo & Dark red & Triangular \\
\hline & & 18 & C. baccatum & Puca Limo & Dark red & Triangular \\
\hline & & 40 & C. chinense & Aji Dulce Rojo & Red & Bell shaped \\
\hline & & 72 & C. chinense & Aji Dulce Rojo & Clear red & Elongated \\
\hline & & 193 & C. baccatum & Escabeche & Orange & Elongated \\
\hline & & 10ica & C. chinense & Miscucho & Lemon yellow & Triangular \\
\hline & & 11 ica & C. chinense & Corazon de Paloma Rojo & Red & Triangular \\
\hline & & $12 p$ & C. frutescens & Charapita Rojo & Red & Almost round \\
\hline & & lica & C. frutescens & Charapon & Red & Almost round \\
\hline & & 5ica & C. baccatum & Ayucllo & Clear red & Bell shaped \\
\hline & & 6ica & C. baccatum & Challuaruro & Clear red & Elongated \\
\hline & & 7 ica & C. chinense & Limo & Red & Triangular \\
\hline
\end{tabular}

\section{Conclusions}

The present study investigated the agro-morphological characteristics of 31 native Peruvian chili peppers. These peppers showed a great diversity in fruit length, fruit diameter, fruit weight, number of fruits per plant, plant height and color. Various shades of red and yellow, along with colors like orange and brown, were present. The sensory properties of these 31 native Peruvian chili peppers were determined by DSA. This is the first detailed study on the sensory attributes of fresh chili peppers from Peru, a country with a long history in the culinary use of these spices. The results from the DSA differentiated the 31 chili peppers into six groups based on their sensory attributes. The majority of them (12 out of 31) had a predominant bell pepper attribute (groups D, E and F), while the remaining was either fruity (groups A and C) or herbal (group B). Each group included different Capsicum species with different agro-morphological characteristics, indicating that sensory attribute is not specific to one species or one form of chili pepper. These four flavor (acid, bell-pepper, sweet and tomato) and six aroma (apple, citrus, fruity, herbal, oregano and passion fruit) attributes were all significantly different among the 31 chili peppers. These sensory attributes and overall results will be helpful for producers and the gastronomy sector for the selection of desirable chili peppers in order to increase consumer satisfaction.

\section{Acknowledgments}

The authors are grateful to FONDECYT-CONCYTEC (Contract 206-2013-CONCyTEC-P) and PNICP-Innovate Peru (Contract 128-FINCyT-IA-2013) for their financial support.

\section{References}

[1] Meckelmann, S. W., Riegel, D. W., Van Zonneveld, M. J., Ríos, L., Peña, K., Ugas, R., Quinonez, L., Mueller-Seitz, E., and Petz, M. 2013. "Compositional Characterization of Native Peruvian Chili Peppers (Capsicum spp.).” J. Agric. Food Chem. 61 (10): 2530-7.

[2] Meckelmann, S. W., Riegel, D. W., Van Zonneveld, M., Ríos, L., Peña, K., Mueller-Seitz, E., and Petz, M. 2015. "Capsaicinoids, Flavonoids, Tocopherols, Antioxidant Capacity and Color Attributes in 23 Native Peruvian Chili Peppers (Capsicum spp.) Grown in Three Different Locations.” Eur. Food Res. Technol. 240 (2): 273-83. 
[3] Meckelmann, S. W., Jansen, C., Riegel, D. W., Van Zonneveld, M., Ríos, L., Peña, K., and Petz, M. 2015. "Phytochemicals in Native Peruvian Capsicum pubescens (Rocoto)." Eur. Food. Res. Technol. 241 (6): 817-25.

[4] Patel, K., Ruiz, C., Calderon, R., Marcelo, M., and Rojas, R. 2016. "Characterization of Volatile Profiles in 50 Native Peruvian Chili Pepper Using Solid Phase Microextraction-Gas Chromatography Mass Spectrometry (SPME-GCMS).” Food Res. Int. 89: 471-5.

[5] Luning, P. A., De Vries, R. V., Yuksel, D., Ebbenhorst-Seller, T., Wichers, J. J., and Roozen, J. P. 1994. "Combined Instrumental and Sensory Evaluation of Flavor of Fresh Bell Peppers (Capsicum annuum) Harvested at Three Maturation Stages." J. Agric. Food Chem. 42 (12): 2855-61.

[6] Garruti, D. A., Pinto, N. O. F., Alves, V. C. C., Da Penha, M. F. A., Tobaruela, E. C., and Araújo, I. M. S. 2013. "Volatile Profile and Sensory Quality of New Varieties of Capsicum chinense Pepper." Ciênc. Tecnol. Aliment. 33: 102-8.

[7] Kollmannsberger, H., Rodríguez-Burruezo, A., Nitz, S., and Nuez, F. 2011. "Volatile and Capsaicinoid Composition of Ají (Capsicum baccatum) and Rocoto (Capsicum pubescens), Two Andean Species of Chili Peppers.” J. Sci. Food Agric. 91 (9): 1598-611.

[8] Eggink, P. M., Maliepaard, C., Tikunov, Y., Haanstra, J. P., Bovy, A. G., and Visser, R. G. 2012. "A Taste of Sweet Pepper: Volatile and Non-volatile Chemical Composition of Fresh Sweet Pepper (Capsicum annuum) in Relation to Sensory Evaluation of Taste." Food Chem. 132 (1): 301-10.

[9] International Plant Genetic Resources Institute (IPGRI). 1995. Descriptors for Capsicum (Capsicum spp.). Rome, Italy: IPGRI. (in Spanish)

[10] Commission for Technical and Commercial Regulations. 2009. NTP-ISO 6564: Sensory Analysis. Methodology to Establish the Flavor Profile, Commission for Technical and Commercial Regulations, Lima. (in Spanish)

[11] Commission for Technical and Commercial Regulations. 2008. NTP-ISO 6658: Sensory Analysis. Methodology to Establish the Flavor Profile, Commission for Technical and Commercial Regulations, Lima. (in Spanish) 